\title{
Elementos técnicos para un necesario debate sobre mediciones de concentración de ingresos en Colombia
}

\section{Technical elements for a necessary debate on income concentration measurements in Colombia}

Luis Jorge Garay S.* y Jorge Enrique Espitia Z.***
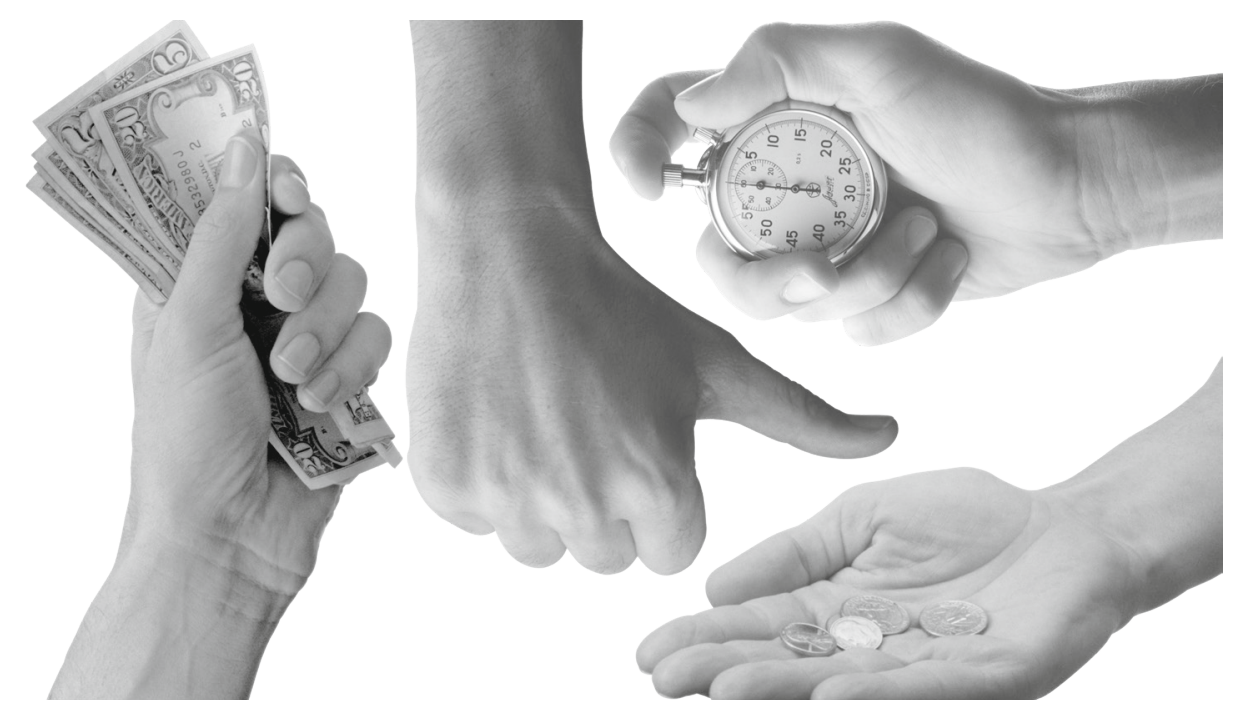

\section{Resumen}

El objetivo de este trabajo es aportar elementos sobre un debate "tabú", hace unos años, pues hablar de desigualdad era fomentar la "lucha de clases". La fuente de información es la Encuesta nacional de presupuestos de hogares y las declaraciones fiscales del impuesto a la renta. La combinación de estas dos fuentes de información es necesaria para realizar una aproximación a la distribución del ingreso en Colombia, considerado el tercer país con mayor desigualdad en el mundo. De igual manera, se observan unos altos niveles de concentración del ingreso, así como una clase media con una alta probabilidad de caer en la pobreza dado el confinamiento, pues el $42 \%$ de esta tiene un ingreso promedio entre 2 y 3 salarios mínimos legales vigentes.

Palabras clave: distribución del ingreso, desigualdad, lucha de clases.

Citar este artículo como: Garay, L. J. y Espitia, J. E. (2020). Elementos técnicos para un necesario debate sobre mediciones de concentración de ingresos en Colombia. Revista Papeles, 12(23), 19-38.

Fecha de recibido: junio 1 del 2020Fecha de aprobado: julio 15 de 2020

* Ingeniero industrial de la Universidad de los Andes, MA en economía de la misma universidad y Ph. D. del MIT. Correo electrónico: ljgara@yahoo.com.mx

*: Ingeniero eléctrico de la ECI, economista de la UNAL. Correo electrónico: jeespitia@gmail.com 


\begin{abstract}
The objective of this work is to contribute elements about a "taboo" debate, a few years ago, since to speak of inequality was to promote the "class struggle". The source of information is the Encuesta nacional de presupuestos de hogares and income tax returns. The combination of these two sources of information is necessary to make an approximation to the distribution of income in Colombia, considered the third most unequal country in the world. Similarly, high levels of income concentration are observed, as well as a middle class with a high probability of falling into poverty given the confinement, since $42 \%$ of it has an average income between 2 and 3 current legal minimum wages.
\end{abstract}

Keywords: inequality, income concentration, class struggle.

\title{
Introducción
}

El objetivo de este documento de trabajo es el de aportar elementos técnicos sobre un debate de importancia como lo es la desigualdad, la concentración del ingreso y sus fuentes de medición. Tal y como lo plantea Goldhammer (2018), este era un tema "tabú" hace unos años, pues,

hablar de desigualdad resultaba tan polémico que quienes pretendían iniciar una conversación sobre estas cuestiones eran acusados de fomentar la "lucha de clases". De hecho, había quienes justificaban la desigualdad como un incentivo para el esfuerzo, la innovación y el desarrollo económico (p. 55),

buscando olvidar aspectos tales como las posibles distorsiones en los "procesos políticos democráticos" (p. 61) y, así, romper el cordón umbilical al buscar no "considerar el rol del poder y la política como factores generadores de la desigualdad" (Derennoncourt, 2018, p. 721).

En el documento se presentan los resultados de la concentración del ingreso a partir de dos fuentes. Una, la Encuesta nacional de presupuestos de hogares (ENPH) 2016 y 2017 realizada por el DANE sobre un total de 87.201 hogares encuestados.

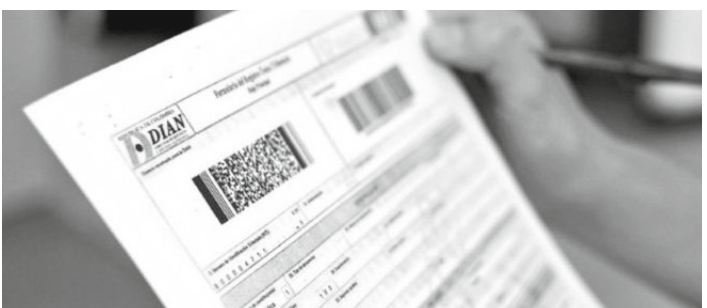

La información de la ENPH se recolectó en 13 ciudades y sus áreas metropolitanas, 11 ciudades intermedias, 8 capitales de departamentos de la Amazonía y Orinoquía y 6 municipios representativos por sí mismos. Igualmente en cabeceras, centros poblados y rurales dispersos en aproximadamente 130 municipios del país. Esta etapa tuvo lugar desde el 11 de julio de 2016 hasta el 9 de julio de $2017^{1}$.

Y dos, los resultados de las declaraciones de renta y complementarios para las personas naturales publicados por la DIAN.

De igual manera, se presentan los resultados de concentración del ingreso, en términos de la clasificación de los hogares adoptada por el DANE en cuatro clases sociales: pobres, vulnerables, clase media y alta.

1 DANE. (2018). "Ficha metodológica Encuesta Nacional de Presupuestos de los Hogares -ENPH". Código: DSO-ENPHFME-001. Versión: 1. Fecha: 2/Ago. /2018. 


\section{Antecedentes: objetivos de la Encuesta nacional de presupuestos de hogares (ENPH) 2016 y 2017}

El objetivo general de la ENPH del DANE es el de "Obtener información sobre el monto y la distribución de los gastos de los hogares colombianos, así como el monto y las fuentes de los ingresos", para, así, “Conocer las características y patrones de consumos de los hogares" $y$, en consecuencia, "Obtener la información requerida para estimar las líneas de pobreza extrema y de pobreza del país", de tal forma que "permita caracterizar la distribución del ingreso del hogar asociada a las características demográficas, educativas y económicas de las personas del hogar" (DANE, 2018).

Esta encuesta es la base de información básica que sirve para el análisis empírico desarrollado en el presente ensayo.

\section{La concentración del ingreso total}

De acuerdo con el DANE, el ingreso del hogar comprende las "entradas en efectivo, en especie o en servicios que por lo general son frecuentes y regulares, destinadas al hogar o a los miembros del hogar por separado y se reciben con intervalos anuales o con mayor frecuencia", en el entendido de que "tales entradas están potencialmente disponibles para el consumo efectivo". De igual manera, se define el ingreso disponible como el "Ingreso del hogar menos ciertos gastos en impuestos de renta y patrimonio, contribuciones a la seguridad social, transferencias a otros hogares, gobierno e instituciones sin ánimo de lucro e intereses y rentas de la tierra" (DANE, 2020, p. 28) ${ }^{2}$.

El cálculo del índice de concentración Gini para el total nacional, cabeceras y centros poblados y rural disperso se obtiene con base en los cuadros de salida publicados por el DANE (cuadro 1). La ENPH muestra que el decil más alto de ingresos concentra una elevada proporción del ingreso total (39\%) y que conjuntamente con el decil 9 alcanzan

2 DANE (2020). Encuesta nacional de presupuestos de los hogares (metodología general) ENPH. Código: Dso-Enph-Met-001 Versión: 2 Fecha: 24Feb-2020. Proceso: Procesos misionales operación o investigación estadística: ENPH - Encuesta nacional. una participación de hasta el 55\%. A su vez, la relación de ingresos totales del decil 10 con respecto a los del decil 1 llega a 34 .

Cabe recordar que, según las declaraciones de renta ante la DIAN para el año 2017, el ingreso bruto declarado por las personas naturales del decil 10 es 61 veces superior al del decil 1, y los porcentajes de concentración del decil 9 y 10 fueron de $12.2 \%$ y $51.0 \%^{3}$, respectivamente; de lo que se puede deducir una subrepresentación de los deciles altos de ingreso en la ENPH respecto a las declaraciones del impuesto sobre la renta y complementarios.

De otro lado, la encuesta muestra que el nivel de dispersión de los ingresos en el decil 10 es superior al del resto de deciles, de ahí la necesidad de examinar con mayor detalle su distribución entre subdeciles del decil 10. En efecto, el nivel de dispersión del decil 1 es de 89 millones de pesos, mientras que el del decil 10 es, aproximadamente, 4 mil millones de pesos, equivalente a 2.6 veces la dispersión media.

3 De acuerdo con las declaraciones de renta y complementarios de las personas naturales obligadas a llevar contabilidad para el año gravable 2004, la relación entre el ingreso bruto del decil 10 y el decil 1 fue de 225; mientras para las personas naturales no obligadas a llevar contabilidad fue de 70. Esta misma relación para el año 2016 fue de 284 y 47 , respectivamente. 
Cuadro 1. Distribución de ingreso total de hogares por deciles de ingreso según cabeceras, centros rurales y total nacional (2016-2017)

\begin{tabular}{|c|c|c|c|c|c|c|}
\hline & \multicolumn{2}{|c|}{ Total nacional } & \multicolumn{2}{|c|}{ Cabeceras } & \multicolumn{2}{|c|}{ Centros poblados y rural disperso } \\
\hline & $\begin{array}{c}\text { hogares } \\
\text { (miles) }\end{array}$ & $\begin{array}{l}\text { Ingreso total } \\
\text { (MM \$/Mes) }\end{array}$ & $\begin{array}{c}\text { Total de } \\
\text { hogares } \\
\text { (miles) }\end{array}$ & $\begin{array}{l}\text { Ingreso total } \\
(\mathrm{MM} \text { \$/Mes) }\end{array}$ & $\begin{array}{c}\text { Total de } \\
\text { hogares } \\
\text { (miles) }\end{array}$ & $\begin{array}{l}\text { Ingreso total } \\
\text { (MM \$/Mes) }\end{array}$ \\
\hline Total & 14.350 & 32.297 & 11.277 & 28.702 & 3.073 & 3.595 \\
\hline Decil 1 & 1.435 & 375 & 1.127 & 354 & 307 & 58 \\
\hline Decil 2 & 1.435 & 830 & 1.128 & 795 & 307 & 115 \\
\hline Decil 3 & 1.435 & 1.168 & 1.127 & 1.082 & 307 & 157 \\
\hline Decil 4 & 1.435 & 1.484 & 1.128 & 1.362 & 307 & 200 \\
\hline Decil 5 & 1.435 & 1.833 & 1.128 & 1.685 & 307 & 242 \\
\hline Decil 6 & 1.435 & 2.275 & 1.128 & 2.067 & 307 & 288 \\
\hline Decil 7 & 1.435 & 2.842 & 1.127 & 2.546 & 307 & 343 \\
\hline Decil 8 & 1.435 & 3.651 & 1.128 & 3.257 & 308 & 419 \\
\hline Decil 9 & 1.435 & 5.108 & 1.128 & 4.527 & 306 & 552 \\
\hline Decil 10 & 1.436 & 12.730 & 1.128 & 11.027 & 308 & 1.222 \\
\hline Gini & & 0,54370 & & 0,53027 & & 0,47317 \\
\hline D10/D1 & & 34 & & 31 & & 21 \\
\hline
\end{tabular}

Fuente:DANE.https://www.dane.gov.co/index.php/estadisticas-por-tema/pobreza-y-condiciones-de-vida/encues ta-nacional-de-presupuestos-de-los-hogares-enph

Ello se ve reflejado en elevados índices Gini de concentración de ingresos totales de hogares que oscilan entre uno de 0.5437, para el promedio nacional, en comparación con unos de 0.5303, para cabeceras municipales, y hasta uno de 0.4732 , para centros poblados y rural disperso.
Así, entonces, se corrobora por qué Colombia es quizás el segundo o tercer país en la región con mayor nivel de desigualdad de ingresos, además de constatarse que la desigualdad en cabeceras municipales es ostensiblemente mayor que en los centros rurales, en los que también subsisten mayores niveles de pobreza multidimensional, de informalidad y de precariedad institucional (Garay y Espitia, 2019, cap. 4).

\section{La concentración del ingreso corriente monetario}

El ingreso corriente monetario lo conforman rubros tales como:

(i) La remuneración monetaria por trabajo en condición de asalariado (sueldos y salarios, horas extras, auxilios y subsidios laborales, gastos de representación, primas y bonificaciones mensuales, primas y bonificaciones anuales); (ii) El ingreso monetario por trabajo independiente (ganancia neta -ingreso empleo independiente áreas urbanas para el caso de hogares en cabeceras; ganancia neta- ingreso empleo independiente para el caso de áreas rurales);

(iii) Otros ingresos monetarios provenientes del trabajo (ingresos por empleo secundario, 
ingresos de trabajo para personas desocupadas, ingresos de trabajo para personas inactivas);

(iv) Renta de la propiedad (arriendos);

(v) Otros ingresos corrientes y transferencias (ayudas, intereses y cesantías, pensiones) (DANE. 2020. p. 7).

La relación promedio entre el ingreso monetario y el ingreso total es de $85 \%$. Entre los componentes más importantes del ingreso monetario se encuentran el de trabajo (48\%), el obtenido a través del trabajo independiente
(26\%), así como el recibido por transferencias (15\%).

La heterogeneidad entre deciles es una norma, en especial entre los deciles de bajos ingresos $(1,2$ y 3$)$ y los de altos ingresos $(8,9$ y 10), así como las diferencias existentes entre el decil más pobre y el más rico. Por ejemplo, los ingresos por trabajo principal de los asalariados del decil 10 es 361 veces superior a los del decil 1 a nivel nacional, de 147 en el caso de las cabeceras y de 246 en el de los centros poblados y el rural disperso (cuadro 2).

Cuadro 2. Composición del Ingreso corriente monetario mensual de las unidades de gasto 2016-2017

\begin{tabular}{|c|c|c|c|c|c|c|c|c|c|}
\hline & 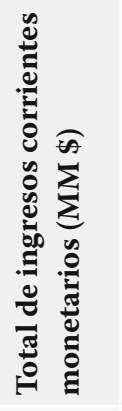 & 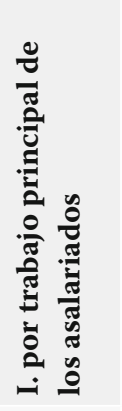 & 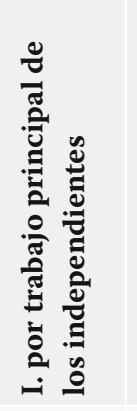 & 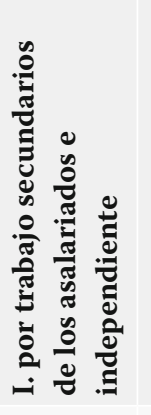 & 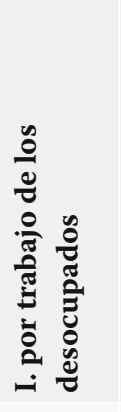 & 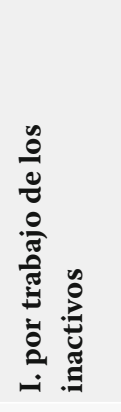 & 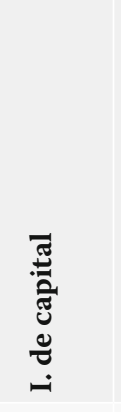 & 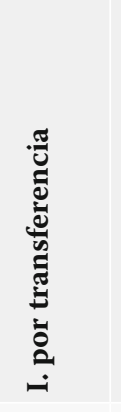 & 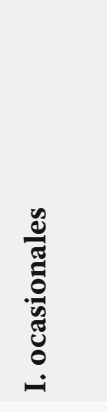 \\
\hline Total & 27.544 & $48,0 \%$ & $26,1 \%$ & $1,5 \%$ & $0,7 \%$ & $0,5 \%$ & $5,4 \%$ & $15,0 \%$ & $2,8 \%$ \\
\hline 1 & 173 & $8,6 \%$ & $42,9 \%$ & $1,4 \%$ & $1,8 \%$ & $0,9 \%$ & $3,9 \%$ & $37,1 \%$ & $3,3 \%$ \\
\hline 2 & 568 & $18,6 \%$ & $53,2 \%$ & $1,5 \%$ & $1,9 \%$ & $0,9 \%$ & $4,8 \%$ & $17,4 \%$ & $1,7 \%$ \\
\hline 3 & 900 & $27,4 \%$ & $47,0 \%$ & $1,3 \%$ & $1,4 \%$ & $0,6 \%$ & $3,3 \%$ & $17,6 \%$ & $1,3 \%$ \\
\hline 4 & 1.177 & $45,6 \%$ & $35,2 \%$ & $0,8 \%$ & $1,4 \%$ & $0,7 \%$ & $2,4 \%$ & $12,9 \%$ & $1,0 \%$ \\
\hline 5 & 1.466 & $46,0 \%$ & $32,7 \%$ & $1,1 \%$ & $1,6 \%$ & $0,6 \%$ & $3,4 \%$ & $13,0 \%$ & $1,5 \%$ \\
\hline 6 & 1.874 & $46,7 \%$ & $30,7 \%$ & $1,1 \%$ & $1,1 \%$ & $0,5 \%$ & $4,2 \%$ & $14,3 \%$ & $1,6 \%$ \\
\hline 7 & 2.392 & $53,0 \%$ & $26,7 \%$ & $0,8 \%$ & $1,0 \%$ & $0,5 \%$ & $3,8 \%$ & $12,7 \%$ & $1,4 \%$ \\
\hline 8 & 3.107 & $53,1 \%$ & $25,5 \%$ & $1,1 \%$ & $0,8 \%$ & $0,5 \%$ & $4,3 \%$ & $13,3 \%$ & $1,3 \%$ \\
\hline 9 & 4.442 & $55,2 \%$ & $21,4 \%$ & $1,3 \%$ & $0,6 \%$ & $0,5 \%$ & $4,8 \%$ & $14,1 \%$ & $2,0 \%$ \\
\hline 10 & 11.435 & $47,2 \%$ & $22,1 \%$ & $2,1 \%$ & $0,3 \%$ & $0,5 \%$ & $7,2 \%$ & $16,1 \%$ & $4,4 \%$ \\
\hline Gini & 0,5789 & 0,6240 & 0,4517 & 0,7003 & 0,2731 & 0,5170 & 0,7041 & $\mathbf{0 , 5 8 0 0}$ & 0,7698 \\
\hline D10/D1 & 66 & 361 & 34 & 98 & 11 & 36 & 122 & 29 & 88 \\
\hline \multicolumn{10}{|c|}{ Cabeceras } \\
\hline Gini & 0,5520 & 0,5732 & 0,4388 & 0,7252 & 0,2056 & 0,4775 & 0,6899 & 0,5756 & 0,7801 \\
\hline D10/D1 & 43 & 147 & 19 & 65 & 5 & 18 & 90 & 27 & 87 \\
\hline \multicolumn{10}{|c|}{ Centros poblados y rural disperso } \\
\hline Gini & 0,5064 & 0,6181 & 0,3766 & $\mathbf{0 , 5 8 7 9}$ & 0,4145 & 0,4770 & 0,7678 & 0,4370 & 0,6646 \\
\hline D10/D2 & 30 & 246 & 18 & 39 & 14 & 64 & 224 & 10 & 40 \\
\hline
\end{tabular}

Fuente:DANE.https://www.dane.gov.co/index.php/estadisticas-por-tema/pobreza-y-condiciones-de-vida/encues ta-nacional-de-presupuestos-de-los-hogares-enph 
A su vez, el índice Gini de concentración de ingresos corrientes monetarios de hogares es claramente mayor que el de ingresos totales de hogares, al ser de 0.5789 para el promedio nacional, 0.5520 para cabeceras municipales y 0.5064 para centros poblados y rurales aislados (cuadro 2), en lo que se reitera el patrón diferencial a nivel territorial comentado previamente. Con la característica adicional de que en el caso de los ingresos monetarios corrientes por trabajo de asalariados, como el principal componente de los ingresos corrientes (de un 48.0\%, en promedio), el índice Gini es significativamente mayor hasta en niveles de $0.6240,0,5732$ y 0.6181 , respectivamente, consecuente con un agravamiento de la inequidad en cuanto ingresos salariales de hogares.

Un elemento adicional a destacar del ingreso monetario corresponde a la participación de los ingresos por trabajo principal de los asalariados y del ingreso por trabajo principal de los independientes en los ingresos corrientes monetarios, en la medida en que se presenta una reducción de tal participación del ingreso
Gráfico 1. Participación del Ingreso de asalariados y del Ingreso de los independientes en el ingreso corriente del hogar por deciles 2016-2017 (\%)

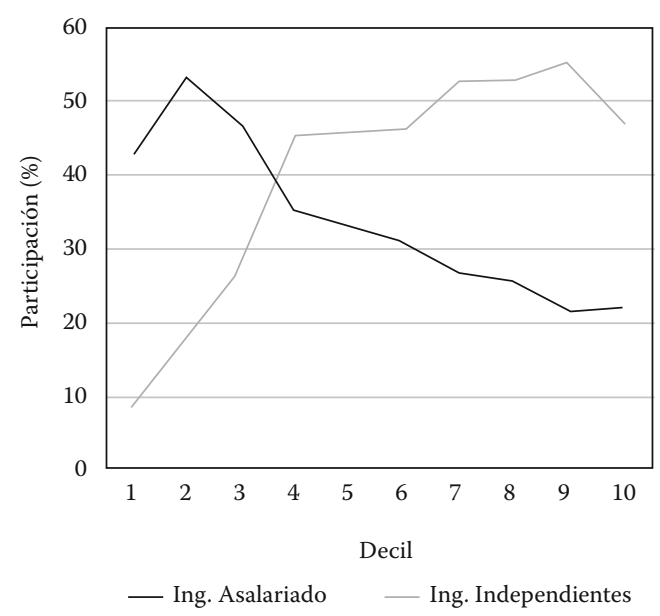

Fuente: DANE, ENPH, cálculos de los autores.

independiente en cuanto se avanza hacia los deciles de mayores ingresos, en sentido contrario a lo que sucede con el ingreso asalariado (gráfico 1).

\section{Concentración del ingreso corriente no monetario}

El ingreso no monetario lo constituyen rubros tales como: el alquiler imputado del servicio de la vivienda ocupada por su propietario, el subsidio de vivienda en especie que recibe el hogar; la diferencia entre valor estimado de alimentos que reciben los niños y las niñas menores de 3 años y el valor mensual pagado por ellos; la diferencia entre el valor estimado de alimentos que reciben las personas que estudian y el valor mensual pagado por ellas; otros subsidios del gobierno en especie que recibe el hogar; el valor estimado de bienes y servicios adquiridos por el hogar por autoconsumo, autosuministro y pago en especie, entre otros (DANE, 2020, p. 7).

El alquiler imputado del servicio de la vivienda ocupada por su propietario es uno de los rubros más importantes del ingreso corriente no monetario del hogar. Se estima a través de la siguiente pregunta: "Si tuviera que pagar arriendo por esta vivienda, ¿cuánto estima que tendría que pagar mensualmente?". El DANE es claro en señalar que "cuando el hogar reside en una vivienda propia no paga arriendo por vivir allí, pero la vivienda le está prestando un servicio que de otra forma tendría que pagar (...); este servicio debe ser valorado por la persona propietaria". A este valor se le denomina "Alquiler imputado de los servicios de la vivienda propia usada por su propietario", el cual se toma como ingreso, en razón de que la vivienda es parte del capital del hogar y el arriendo constituye la renta proveniente de dicho capital. Cuando la vivienda es cedida en forma gratuita por una entidad u hogar particular, sin vínculo laboral entre 
Cuadro 3. Distribución del valor de alquiler imputado y su participación en los ingresos totales por hogar según decil de ingreso 2016-2017

\begin{tabular}{|c|c|c|c|}
\hline Decil & $\begin{array}{l}\text { P5110* fac. exp. } \\
\text { (MM\$) }\end{array}$ & $\begin{array}{c}\text { Distribuc. } \\
\text { P5110* fac. exp. }\end{array}$ & $\begin{array}{c}\text { Participac. } \\
\text { P5110* fac. exp./Ingr. }\end{array}$ \\
\hline 1 & 112 & $3 \%$ & $30 \%$ \\
\hline 2 & 174 & $5 \%$ & $21 \%$ \\
\hline 3 & 156 & $4 \%$ & $13 \%$ \\
\hline 4 & 202 & $5 \%$ & $14 \%$ \\
\hline 5 & 258 & $7 \%$ & $14 \%$ \\
\hline 6 & 292 & $8 \%$ & $13 \%$ \\
\hline 7 & 344 & $9 \%$ & $12 \%$ \\
\hline 8 & 430 & $11 \%$ & $12 \%$ \\
\hline 9 & 587 & $16 \%$ & $11 \%$ \\
\hline 10 & 1.219 & $32 \%$ & $10 \%$ \\
\hline Total & 3.779 & $100 \%$ & $12 \%$ \\
\hline D10/D1 & 11 & & \\
\hline \multicolumn{4}{|c|}{ Fuente: DANE, ENPH, cálculos de los autores. } \\
\hline \multicolumn{4}{|c|}{$\begin{array}{l}\text { ellos, o esta es ocupada sin consentimiento } \\
\text { del dueño, la autovaloración se considera } \\
\text { como una transferencia y bajo este concepto } \\
\text { entra a formar parte del ingreso corriente del } \\
\text { hogar. Si el hogar recibe la vivienda como } \\
\text { parte de salario, el valor estimado sobre su } \\
\text { alquiler es un ingreso laboral y se toma como } \\
\text { salario en especie (DANE, 2020, p. 4). }\end{array}$} \\
\hline \multicolumn{4}{|c|}{$\begin{array}{l}\text { De acuerdo con los microdatos de la ENPH } \\
\text { y el factor de expansión, se encuentra que el } \\
\text { valor estimado de este rubro de alquiler es } \\
\text { de } \$ 3.8 \text { billones, de los cuales el } 32 \% \text { se con- } \\
\text { centra en el decil } 10 \text {, en tanto que la relación } \\
\text { para tal rubro entre el decil } 10 \text { y el decil } 1 \text { es } \\
\text { de } 11 \text { veces. Este rubro corresponde hasta el } \\
30 \% \text { del ingreso total de hogares del decil } 1 \text { y } \\
\text { el } 10 \% \text { del ingreso total de hogares del decil } \\
10 \text { (cuadro } 3 \text { ). }\end{array}$} \\
\hline $\begin{array}{l}\text { La distribuci } \\
\text { tancia en los } \\
\text { según decile } \\
\text { incrementar } \\
\text { del ingreso to }\end{array}$ & $\begin{array}{l}\text { On del valor del rubro y su impor- } \\
\text { ingresos totales de los hogares } \\
\text { de ingreso parecería tender a } \\
\text { el índice de concentración Gini } \\
\text { tal de hogares. }\end{array}$ & & \\
\hline
\end{tabular}




\section{Concentración del ingreso corriente monetario disponible personal}

El ingreso corriente monetario disponible es el ingreso corriente monetario menos los descuentos y deducciones obligatorias, entre las cuales se encuentran: descuento por salud, impuesto de industria, comercio y avisos -ICA-; impuesto al valor agregado -IVA-; retención en la fuente; fondo de solidaridad pensional; pago a fondos de pensiones. El cuadro de salida que reporta el DANE se encuentra por perceptores de ingreso.
Los índices de participación muestran cómo el ingreso de los perceptores en el decil más rico es 52 veces superior al de los perceptores del decil 1, y el índice de concentración cómo el promedio nacional es relativamente superior al de las cabeceras y al de los centros poblados y rural disperso: 0.6315 vs. 0.6113 y 0.6155 , respectivamente (cuadro 4).

Cuadro 4. Distribución de ingreso corriente disponible total de perceptores según decil de ingresos (2016-2017)

\begin{tabular}{|c|c|c|c|c|c|c|}
\hline & \multicolumn{2}{|c|}{ Total nacional } & \multicolumn{2}{|c|}{ Cabeceras } & \multicolumn{2}{|c|}{$\begin{array}{l}\text { Centros poblados y } \\
\text { rural disperso }\end{array}$} \\
\hline & $\begin{array}{l}\text { Perceptores } \\
\text { (miles) }\end{array}$ & $\begin{array}{c}\text { Ingreso } \\
\text { Corriente } \\
\text { Disponible } \\
\text { (mill \$) }\end{array}$ & $\begin{array}{l}\text { Perceptores } \\
\text { (miles) }\end{array}$ & $\begin{array}{c}\text { Ingreso } \\
\text { Corriente } \\
\text { Disponible } \\
\text { (mill \$) }\end{array}$ & $\begin{array}{l}\text { Perceptores } \\
\quad(\text { miles })\end{array}$ & $\begin{array}{c}\text { Ingreso } \\
\text { Corriente } \\
\text { Disponible } \\
\text { (mill \$) }\end{array}$ \\
\hline Total & 29.198 & 28.017 .465 & 22.671 & 24.988 .129 & 6.527 & 3.029 .336 \\
\hline Decil 1 & 2.919 & 242.251 & 2.266 & 182.130 & 652 & 58.011 \\
\hline Decil 2 & 2.920 & 163.856 & 2.268 & 169.705 & 653 & 26.554 \\
\hline Decil 3 & 2.919 & 463.736 & 2.266 & 551.300 & 653 & 43.050 \\
\hline Decil 4 & 2.921 & 960.682 & 2.268 & 1.032 .352 & 653 & 75.749 \\
\hline Decil 5 & 2.920 & 1.554 .293 & 2.266 & 1.550 .324 & 653 & 134.861 \\
\hline Decil 6 & 2.919 & 2.075 .651 & 2.268 & 1.818 .314 & 652 & 211.441 \\
\hline Decil 7 & 2.920 & 2.471 .097 & 2.267 & 2.108 .830 & 653 & 298.767 \\
\hline Decil 8 & 2.920 & 3.017 .434 & 2.267 & 2.675 .069 & 653 & 402.027 \\
\hline Decil 9 & 2.920 & 4.436 .725 & 2.266 & 3.951 .021 & 652 & 530.831 \\
\hline Decil 10 & 2.920 & 12.631 .741 & 2.268 & 10.949 .085 & 653 & 1.248 .046 \\
\hline Gini & & 0,6315 & & 0,6113 & & 0,6155 \\
\hline D10/D1 & & 52 & & 60 & & 22 \\
\hline
\end{tabular}

Fuente: ENPH. Cálculos propios.

\section{A manera de resumen inicial}

A manera de síntesis, y dados los cambios en términos de índice Gini que existen entre las diferentes variables de ingreso que presenta la Encuesta, en el cuadro 5 se muestra cada uno de ellos, con factores de expansión y sin factores de expansión. Es posible observar cómo la variación del índice de Gini antes y después de impuestos resulta imperceptible (nulo a nivel de los dos primeros decimales): 0.5462 vs. 0.5417 , aparte de ratificarse la alta diferencia existente entre los ingresos según deciles, ya señalada arriba, en la medida en que la relación de ingresos entre el decil $10 \mathrm{y}$ el decil 1 oscila entre 40 y 60 veces sin factor de expansión o entre 33 y 36 con factor de expansión. 
Cuadro 5. Distribución de los diferentes tipos de ingreso reportados por hogar en la ENPH según decil de ingreso en los años 2016 y 2017 (miles de millones)

\begin{tabular}{|c|c|c|c|c|c|c|c|c|}
\hline 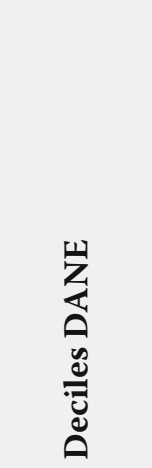 & 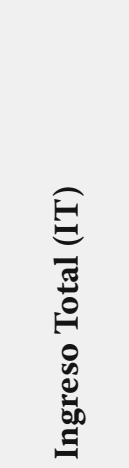 & 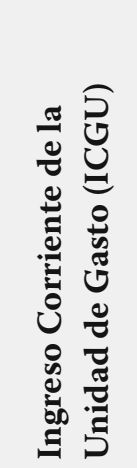 & 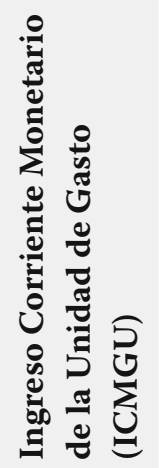 & 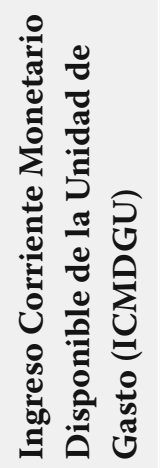 & 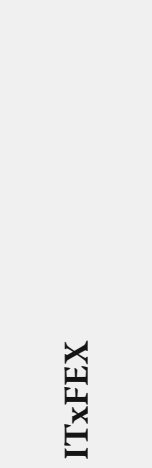 & 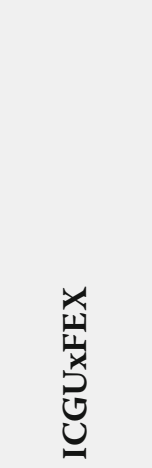 & 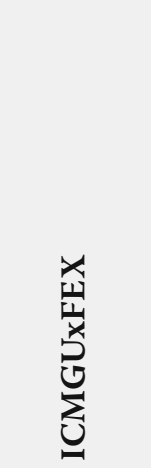 & 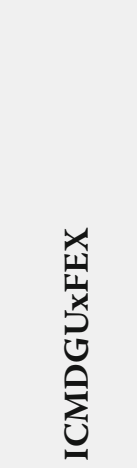 \\
\hline 1 & 1,8 & 1,8 & 1,1 & 1,0 & 375,2 & 369,6 & 235,1 & 226,7 \\
\hline 2 & 4,5 & 4,5 & 3,2 & 3,1 & 848,3 & 839,7 & 613,4 & 603,3 \\
\hline 3 & 6,6 & 6,5 & 5,2 & 5,1 & $1.149,6$ & $1.139,1$ & 921,8 & 896,8 \\
\hline 4 & 8,9 & 8,8 & 7,0 & 6,8 & $1.500,5$ & $1.483,7$ & $1.192,7$ & $1.150,8$ \\
\hline 5 & 11,1 & 11,0 & 8,8 & 8,5 & $1.816,7$ & $1.794,2$ & $1.444,7$ & $1.384,6$ \\
\hline 6 & 14,4 & 14,3 & 11,7 & 11,3 & $2.275,4$ & $2.246,4$ & $1.842,9$ & $1.766,2$ \\
\hline 7 & 19,0 & 18,9 & 15,8 & 15,1 & $2.844,8$ & $2.815,7$ & $2.355,5$ & $2.248,9$ \\
\hline 8 & 24,4 & 24,1 & 20,5 & 19,5 & $3.659,7$ & $3.608,6$ & $3.045,4$ & $2.894,0$ \\
\hline 9 & 34,0 & 33,5 & 29,0 & 27,5 & $5.099,8$ & $5.016,0$ & $4.292,2$ & $4.066,0$ \\
\hline 10 & 74,2 & 71,5 & 63,9 & 60,5 & $12.727,0$ & $12.225,0$ & $10.841,6$ & $10.209,6$ \\
\hline Total & 199,0 & 194,9 & 166,3 & 158,4 & $32.297,0$ & $31.538,0$ & $26.785,2$ & $25.447,0$ \\
\hline D10/D1 & 41 & 40 & 60 & 59 & 34 & 33 & 46 & 45 \\
\hline Gini & 0,5233 & 0,5179 & 0,5462 & 0,5417 & 0,5438 & 0,5384 & 0,5633 & 0,5581 \\
\hline
\end{tabular}

Fuente: ENPH. Cálculos propios.

\section{Distribución del ingreso entre grupos sociales según la ENPH: pobres, vulnerables, clase media y clase alta}

Con base en la propuesta de López-Calva y Ortiz-Juárez (2012), el DANE clasifica en cuatro clases sociales los hogares: i) pobres: hogares con un ingreso diario per cápita por debajo de la línea de pobreza (US\$ 4,06 PPA); ii) Vulnerables: hogares con un ingreso diario per cápita entre la línea de pobreza (LP) y US\$ 10 PPA; iii) Clase Media: hogares con un ingreso diario per cápita entre US\$10 y US\$ 50 PPA, y iv) clase alta: hogares con un ingreso mayor a US\$ 50 PPA al día.

4 Más precisamente, la línea de pobreza oficial.
El DANE en su Boletín Técnico sobre Pobreza Monetaria para el año 2018, señala que si el ingreso per cápita mensual de la unidad de gasto es inferior a $\$ 117.605$ se cataloga en condición de pobreza extrema, y si es inferior a $\$ 257.433$ se clasifica como pobre. "De acuerdo con lo anterior, si un hogar está compuesto por 4 personas, será clasificado como pobre si el ingreso total del hogar está por debajo de \$1.029.732", y en pobreza extrema si el ingreso total del hogar es inferior a $\$ 470.420$ al mes (pp. 2-4). 
Así pues, la clasificación aquí expuesta, y mediante la cual se presentan los resultados de la ENPH, corresponde a: i) hogares en pobreza extrema si el ingreso mensual es inferior a $\$ 470.420$, ii) hogares en pobreza si el ingreso mensual del hogar se encuentra entre $\$ 470.420$ y \$1.029.732; iii) hogares vulnerables, con un ingreso diario per cápita por encima de la línea de pobreza monetaria definida por el DANE, pero por debajo del umbral de US\$10 PPA; iv) hogares de clase media, cuyo ingreso diario per cápita está en el rango de USD $\$ 10$ a USD\$50 PPA, y v) hogares de clase alta, con un ingreso per cápita superior a USD\$50 PPA al día.

López-Calva y Ortiz-Juárez proponen una definición para identificar la clase media con base en "un enfoque de vulnerabilidad a la pobreza". La definición consiste en que quienes han logrado salir de la pobreza, pero cuya situación económica es de inseguridad, tienen una baja probabilidad de "emprender, crear, o demandar cierto patrón de consumo y reafirmar su estatus". Aspecto relevante en estos tiempos de pandemia y pospandemia, cuando la tasa de desempleo ha aumentado de manera significativa y el ingreso de los trabajadores independientes se ha reducido

\section{López-Calva y Ortiz-Juárez proponen} una definición para identificar la clase media con base en "un enfoque de vulnerabilidad a la pobreza". La definición consiste en que quienes han logrado salir de la pobreza, pero cuya situación económica es de inseguridad, tienen una baja probabilidad de "emprender, crear, o demandar cierto patrón de consumo y reafirmar su estatus". drásticamente, al punto que diversos sectores de la opinión pública han tenido que llamar la atención del gobierno nacional para que se implanten programas de intervención social a favor del grupo de hogares vulnerables y pobres, debido a que la pandemia y la estrategia del confinamiento han propiciado que buen número de familias cayeran en vulnerabilidad o incluso en pobreza, puesto que con anterioridad a la pandemia existían cerca de 2.500.000 hogares con un ingreso mensual entre 2 y 3 (salario mínimos legales vigentes) con una alta dependencia del trabajo independiente $y$, en su mayoría, trabajadores no calificados, lo cual los coloca en una situación de inestabilidad e inseguridad laboral; y otros 2.921.000 mil hogares vulnerables con ingreso mensual entre 1 y 2 SMLV, con una alta probabilidad de caer en pobreza ${ }^{5}$. De acuerdo con el estimativo de López-Calva y Ortiz-Juárez para Chile, Perú y México, esta probabilidad de caer en situación de vulnerabilidad sería superior al $25 \%$.

Una vez se clasifican los hogares en Colombia con base en la metodología de López-Calva y Ortiz-Juárez, se encuentra que el periodo de 2016-2017:

(i) El 55\% de los hogares se clasifican en situación de pobreza extrema, pobreza y vulnerabilidad (cuadro 6).

(ii) Los hogares en vulnerabilidad serían cerca de 2.921 .000 y tienen un ingreso mensual promedio igual a 1.6 SMLV.

5 En la medida en que un grupo importante de ciudadanos se ganan la vida vendiendo sus servicios laborales con contratos de trabajo precarios o de manera informal, hay necesidad de "pensar en soluciones innovadoras, como por ejemplo un impuesto negativo a la renta, un complemento salarial o una renta básica. Esto serviría para garantizar un nivel de vida mínimo a los ciudadanos, más allá de su situación laboral y de la compensación que obtienen por su trabajo" (Tyson, L. y Spence, M., 2018, p. 301). 
Cuadro 6. Distribución de hogares y sus ingresos por clases en Colombia 2016-2017

\begin{tabular}{|l|r|r|r|} 
& Personas & Ingreso total $(\mathbf{S})$ & \multicolumn{1}{c|}{ Hogares } \\
\hline Alta & 1.707 .980 & 7.344 .915 .533 .295 & 498.439 \\
Clase media & 22.006 .271 & 18.139 .270 .453 .649 & 5.930 .943 \\
Vulnerable & 9.849 .381 & 3.761 .350 .157 .439 & 2.920 .976 \\
Pobreza & 10.283 .779 & 2.613 .516 .598 .345 & 3.427 .211 \\
Pobreza extrema & 3.831 .975 & 438.121 .979 .409 & 1.572 .834 \\
Total & $\mathbf{4 7 . 6 7 9 . 3 8 6}$ & $\mathbf{3 2 . 2 9 7 . 1 7 4 . 7 2 2 . 1 3 7}$ & $\mathbf{1 4 . 3 5 0 . 4 0 3}$ \\
Gini & $\mathbf{0 . 4 0 8}$ & & $\mathbf{0 . 4 4 7}$
\end{tabular}

Fuente: DANE. Cálculos propios.

(iii) Los hogares de la denominada clase media alcanzarían cerca de 5.931.000, mientras que los de clase alta no superarían 500.000.

(iv) Los hogares de clase alta concentrarían cerca del $23 \%$ de los ingresos totales de los hogares en el país, oscilando entre 10 SMLV y 372 SMLV por hogar al mes; mientras que los hogares de clase media participarían con cerca del $56 \%$ de los ingresos totales, variando su ingreso mensual por hogar entre 2 SMLV y 10 SMLV.

(v) El 42\% de los hogares de la denominada clase media tiene un ingreso mensual que oscila entre los 2 y $3 \mathrm{SMLV}$, altamente concentrado en remuneraciones no salariales y en labores informales, por lo cual es muy probable que la pandemia los hubiera llevado a una situación de vulnerabilidad o de pobreza.

(vi) El ingreso por persona de los hogares de clase media sería, en promedio, de $\$ 823.000$ por mes, lo que ratifica su situación de fragilidad frente a un fenómeno social como la pandemia del Covid-19.

En las dos últimas características reside la razón por la cual la definición de clase media resulta inconvenientemente laxa para propósitos de servir de base para un robusto diseño de política pública social, pues incluye una importante proporción de hogares con una elevada fragilidad tanto por su modesto nivel de ingresos como por sus precarias condiciones laborales, que los hacen susceptibles de caer incluso hasta la condición de pobreza ante un ciclo recesivo de la economía como el reproducido con la pandemia del Covid-19.

(v) El índice de concentración Gini para el conjunto de las cinco clases o conglomerados de hogares es de 0.408 en el caso de personas y de 0.447 en el de hogares.

(vi) Al dividir en subdeciles la clase alta se encuentra que los subdeciles 8 (11\%), 9 (13\%) y $10(27 \%)$ de la clase alta concentran el 51\%, mientras que los subdeciles 1,2 y 3 participan con tan solo un $18 \%$. El índice de concentración Gini del ingreso al interior de la clase alta es de 0.300 (cuadro 7).

(vii) En el caso de la clase media, el subdecil 10 que tiene un ingreso promedio de 6.300 .000 pesos mensuales, concentra el $21 \%$ de los ingresos totales de esta clase. Los subdeciles 1, 2, 3, 4 y 5 tienen un ingreso inferior a 2.500.000 pesos mensuales y concentran el 33\% del ingreso total. Cabe señalar que el ingreso promedio de la clase vulnerable es de 1.300 .000 pesos mensuales. Es por ello que se observa una alta probabilidad que con el confinamiento un sector importante de la denominada clase media entre a engrosar los hogares tanto vulnerables como de pobreza. Este fenómeno fue comparado con la siguiente frase: clase media, bienvenida a la pobreza, en un titular de un diario mexicano (Expansion. com) a mediados de septiembre. 
Cuadro 7. Distribución y concentración de ingreso por subdeciles de la clase alta y media según la ENPH 2016-2017

\begin{tabular}{|c|c|c|c|}
\hline Subdeciles & Hogares & Ingreso total (MM\$) & Ingreso total / hogar \\
\hline Alta & 498.439 & 7.345 & 14.735 .836 \\
\hline 1 & 49.819 & 406 & 8.145 .079 \\
\hline 2 & 49.422 & 429 & 8.688 .512 \\
\hline 3 & 49.768 & 465 & 9.351 .381 \\
\hline 4 & 49.188 & 503 & 10.222 .143 \\
\hline 5 & 49.423 & 543 & 10.985 .939 \\
\hline 6 & 49.794 & 599 & 12.033 .051 \\
\hline 7 & 49.829 & 671 & 13.475 .413 \\
\hline 8 & 49.835 & 777 & 15.582 .459 \\
\hline 9 & 49.478 & 978 & 19.769 .166 \\
\hline 10 & 51.883 & 1.973 & 38.032 .441 \\
\hline Gini & & 0.300 & \\
\hline Clase media & 5.930 .943 & 18.139 & 3.058 .413 \\
\hline 1 & 592.986 & 986 & 1.661 .929 \\
\hline 2 & 593.075 & 1.080 & 1.820 .792 \\
\hline 3 & 593.013 & 1.187 & 2.000 .928 \\
\hline 4 & 592.591 & 1.304 & 2.201 .117 \\
\hline 5 & 593.047 & 1.450 & 2.444 .929 \\
\hline 6 & 593.071 & 1.635 & 2.757 .336 \\
\hline 7 & 592.691 & 1.863 & 3.142 .805 \\
\hline 8 & 593.040 & 2.190 & 3.692 .286 \\
\hline 9 & 591.627 & 2.692 & 4.550 .656 \\
\hline 10 & 595.802 & 3.753 & 6.299 .131 \\
\hline Gini & & 0.264 & \\
\hline
\end{tabular}

Fuente: DANE. ENPH 2016-2017.

\section{Cálculos propios}

A su vez, el índice de Gini de esta clase media es de 0.264 (Cuadro 7).

(viii) Dados los elevados niveles de dispersión del ingreso en el decil 10 de mayores ingresos, resulta necesario analizar su distribución y concentración de ingresos al interior del mismo. Para ello se divide el decil 10 en subdeciles, encontrándose que el ingreso total de los hogares del decil 10 se distribuye de manera aún más desigual entre subdeciles. Así, por ejemplo, el subdecil 1 concentra el 5.1\% del ingreso total del decil 10, mientras que los subdeciles 9 y 10 participan con el $13.9 \%$ y el $28.7 \%$, respectivamente. Así, se destaca que el $1 \%$ de los hogares más ricos en la ENPH llega a concentrar el $11.3 \%$ del ingreso total de los hogares colombianos (cuadro 8) y su ingreso promedio por hogar es 96 veces más grande que el decil de hogares con menores ingresos. Además, el índice de concentración Gini para los hogares del decil 10 -desagregado por 10 subdeciles- es de apenas 0.337 . 
Estos valores contrastan con los encontrados a partir de las declaraciones de renta y complementarios ante la DIAN en 2017, ya que el $1 \%$ de las personas naturales declarantes con mayores ingresos concentran el 25\% del ingreso bruto total declarado en el año 2017 y el ingreso bruto promedio del $1 \%$ más rico fue 294 veces más grande que el declarado por el decil 1.

(ix) En el caso del 1 por 1.000 de los hogares más ricos, se tiene que concentran el 3.3\% del ingreso total de los hogares en el país, el $8.3 \%$ del ingreso de los hogares del decil 10 y el $28.7 \%$ del ingreso de los hogares del subdecil 10. Su ingreso promedio por hogar es 205 veces más alto que el de hogares del decil 1 . Además, al desagregarse el 1\% de los hogares más ricos en 10 subdeciles se registra un índice Gini de concentración de 0.281 , claramente inferior al estimado con base en los ingresos brutos declarados por personas naturales ante la DIAN en $2017^{6}$ (cuadro 9).

Estos valores contrastan con los correspondientes a los de las declaraciones de renta de personas naturales ante la DIAN, dado que el ingreso bruto promedio del 1 por 1.000 de las personas naturales más ricas -las súper superricas- es superior al de las personas del decil 1 en más de 1300 veces. De igual manera, el 1 por 1.000 de las personas naturales más ricas concentra el $11 \%$ del total del ingreso bruto reportado por los más de 3 millones de declarantes en 2017.

En consecuencia, la participación del 1 por 100 y 1 por 1000 de los hogares más ricos del país en el ingreso total de los hogares del país según la ENPH 2016-2017 del DANE es ostensiblemente inferior al equivalente para el caso de las personas naturales declarantes ante la DIAN en 2017. Ello plantea un interrogante serio sobre el grado de representación de este tipo de hogares en la muestra de la ENPH, aparte del nivel de confiabilidad en los datos recolectados en una encuesta como la ENPH sin contrastación con otras fuentes fidedignas, en marcado contraste con el caso de las declaraciones de renta ante la DIAN.

En este punto es de resaltar, como lo señalan Garay y Espitia (2019, capítulo 1), que el

Cuadro 8. Distribución del ingreso de los hogares más ricos (del decil 10) según la ENPH 2016-2017

\begin{tabular}{|l|c|c|c|}
\hline & Hogares & $\begin{array}{c}\text { Ingreso total mensual } \\
\text { (miles de millones de pesos) }\end{array}$ & $\begin{array}{c}\text { Ingreso promedio mensual } \\
\text { (pesos) }\end{array}$ \\
\hline Total nal. & $\mathbf{1 4 . 3 5 0 . 4 0 3}$ & $\mathbf{3 2 . 2 9 7}$ & $\mathbf{2 . 2 5 0 . 6 1 1}$ \\
\hline Subdecil 1 & 143.079 & 654 & 4.573 .479 \\
Subdecil 2 & 142.786 & 695 & 4.868 .213 \\
Subdecil 3 & 143.451 & 750 & 5.230 .033 \\
Subdecil 4 & 143.386 & 809 & 5.644 .346 \\
Subdecil 5 & 143.392 & 892 & 6.217 .359 \\
Subdecil 6 & 142.763 & 986 & 6.904 .098 \\
Subdecil 7 & 143.540 & 1.137 & 7.918 .172 \\
Subdecil 8 & 143.407 & 1.378 & 9.611 .331 \\
Subdecil 9 & 143.297 & 1.770 & 12.352 .141 \\
Subdecil 10 & 146.439 & 3.659 & 24.989 .345 \\
Decil 10 & $\mathbf{1 . 4 3 5 . 5 4 0}$ & $\mathbf{1 2 . 7 3 1}$ & $\mathbf{8 . 8 6 8 . 1 4 0}$ \\
\hline Gini Sub-Decil 10 & & $\mathbf{0 . 3 3 7}$ & \\
\hline
\end{tabular}

Fuente: DANE. ENPH 2016-2017. Cálculos propios.

6 Garay, L. J. y Espitia, J. E. (2019), ib. id., capítulo 3. 
Cuadro 9. Distribución del ingreso del 1 por 1.000 de los hogares más ricos según la ENPH 2016-2017

\begin{tabular}{|l|c|c|c|} 
& Hogares & Ingreso total mes $\mathbf{( M M \$ )}$ & Ingreso promedio mensual (\$) \\
\hline Total nal. & $\mathbf{1 4 . 3 5 0 . 4 0 3}$ & $\mathbf{3 2 . 2 9 7}$ & $\mathbf{2 . 2 5 0 . 6 1 1}$ \\
\hline Subdecil 1 & 14.255 & 211.530 & 14.839 .023 \\
\hline Subdecil 2 & 13.524 & 210.562 & 15.569 .516 \\
\hline Subdecil 3 & 14.345 & 235.304 & 16.403 .206 \\
Subdecil 4 & 14.320 & 253.653 & 17.713 .218 \\
Subdecil 5 & 14.302 & 268.794 & 18.794 .160 \\
Subdecil 6 & 14.306 & 291.927 & 20.405 .907 \\
Subdecil 7 & 13.852 & 319.157 & 23.040 .490 \\
Subdecil 8 & 13.896 & 360.635 & 25.952 .412 \\
Subdecil 9 & 14.052 & 456.498 & 32.486 .337 \\
Subdecil 10 & 19.587 & 1.051 .354 & 53.676 .137 \\
Subdecil 10 & 146.439 & 3.659 .415 & 24.989 .345 \\
Gini Subdecil 10 & & $\mathbf{0 . 2 8 1}$ &
\end{tabular}

Fuente: DANE. ENPH 2016-2017. Cálculos propios.

interés particular por la fiscalidad reside no solamente en la generación de ingresos tributarios y en su impacto en la redistribución, sino porque "constituye una fuente de información para obtener datos exactos sobre el alcance y la naturaleza del capital [...] pero no la única" (Goldhammer, 2018, p. 75) ${ }^{7}$, aunque sí la más comprensiva y detallada en términos de fuentes de ingresos y rentas. De cualquier forma, como afirma Krugman (2018), “(l)as cifras que recaba Hacienda nos dicen mucho sobre las élites económicas" (p. 97)

Por lo anterior, es clara la conveniencia de utilizar los datos tributarios de la DIAN para complementar la información suministrada en la ENPH del DANE y realizar estimativos alternativos sobre la concentración de ingresos de los hogares.

\section{Estimativos alternativos sobre la concentración de ingresos con base en ingresos declarados por personas naturales ante la DIAN}

Hasta ahora se ha observado la existencia de diferencias en índices Gini de concentración del ingreso según la Encuesta nacional de presupuesto de los hogares -ENPH- del DANE y con base en las declaraciones del impuesto sobre la renta y complementarios presentadas por las personas naturales ante la DIAN.

7 Goldhammer, A. (2018). “El fenómeno Piketty". En: Delong, J. B. Boushey, H. y Steinbaum, M. (eds.) (2018). Debatiendo con Piketty. La agenda para la economía y la desigualdad. Ediciones Deusto. Barcelona.
En el caso de las declaraciones de renta y complementarios se observa que el ingreso bruto promedio al mes declarado por el decil 1 es de 861.000 pesos, mientras que el del decil 10 llega a 52.191.000 pesos. La relación de ingresos entre el decil 10 y el decil 1 es de 61 veces, lo que equivale a decir que el ingreso declarado

8 Krugman, P. (2018). "Por qué estamos en la Nueva Edad Dorada". En: Delong, J. B., Boushey, H. y Steinbaum, M. (eds.) (2018). Debatiendo con Piketty. La agenda para la economía y la desigualdad. Ediciones Deusto. Barcelona. 
de las personas naturales más ricas del país es 61 veces superior al del decil 1 (cuadro 10).

En concordancia, el decil 10 declara el 51\% del ingreso bruto total declarado por personas naturales en el país, mientras que el decil 1 tan solo declara el $0.8 \%$. El índice Gini de concentración de ingreso alcanza el 0.6159 en 2017. Este índice promedio, según ingresos declarados ante la DIAN, es sustancialmente mayor que el respectivo promedio de ingresos totales acordes con la ENPH: 0.6159 vs. 0.5437 , pero similar al de ingresos corrientes disponibles según la ENPH: 0.6159 vs. 0.6315 .

Ahora bien, ante la elevada participación en el ingreso declarado total y dada la alta dispersión en los ingresos de las personas naturales más ricas, pertenecientes al decil 10, el cual es 748 veces el correspondiente al decil 1, resulta indispensable estudiar su distribución interna, es decir, a nivel de subdeciles del decil 10.

A nivel de subdeciles del decil 10 se observa que el ingreso promedio del subdecil 10, es decir, del 1 por ciento más rico, es del orden de los 253 millones de pesos mensuales, 294 veces superior al ingreso declarado por el decil 1. Este 1 por ciento más rico concentra el $24.8 \%$ del ingreso total declarado, así como el $48.5 \%$ del ingreso del decil 10. El índice de concentración Gini entre los subdeciles del decil 10 fue de 0.5515 para 2017, significativamente superior al correspondiente según la ENPH -0.5515 vs. $0.337-\mathrm{y}$ algo inferior al índice promedio nacional entre los 10 deciles de ingreso.

Respecto al 1 por 1.000 más rico, perteneciente al subdecil 10 del sub-decil 10 del decil 10, el ingreso promedio es del orden de 1300 veces superior al ingreso declarado por el decil 1. Además, el 1 por 1.000 más rico concentra el $11.0 \%$ del ingreso total declarado por las personas naturales en el país, así como el 44.3\% del ingreso del subdecil 10, lo cual se refleja en un índice Gini de concentración de ingresos declarados ante la DIAN de 0.5183 para ese grupo superrico de personas naturales. Este índice es inferior al índice Gini promedio nacional de ingresos totales según la ENPH para 2017.

Cuadro 10. Distribución del ingreso bruto declarado por las personas naturales ante la DIAN en 2017

\begin{tabular}{|c|c|c|c|c|c|c|c|c|}
\hline \multirow[t]{2}{*}{ Decil } & \multirow[b]{2}{*}{ Declarantes } & \multirow[b]{2}{*}{$\begin{array}{c}\text { Ingreso } \\
\text { Bruto } \\
\text { (MMS) }\end{array}$} & \multirow[b]{2}{*}{$\begin{array}{c}\text { Ingreso } \\
\text { Promedio } \\
\text { Mes (\$) }\end{array}$} & \multirow[b]{2}{*}{$\begin{array}{c}\% \text { en el } \\
\text { Total }\end{array}$} & \multicolumn{2}{|c|}{ Subdecil Decil 10} & \multicolumn{2}{|c|}{ Subdecil 10 Decil 10} \\
\hline & & & & & $\begin{array}{c}\text { Ingreso } \\
\text { Promedio } \\
\text { Mes (\$) }\end{array}$ & $\begin{array}{c}\% \text { en } \\
\text { el } \\
\text { Total }\end{array}$ & $\begin{array}{c}\text { Ingreso } \\
\text { Promedio } \\
\text { Mes (\$) }\end{array}$ & $\begin{array}{c}\% \text { en el } \\
\text { Total }\end{array}$ \\
\hline 1 & 298105 & 3.080 & 861.008 & $0,8 \%$ & 16.782 .029 & $1,6 \%$ & 85.218 .548 & $0,8 \%$ \\
\hline 2 & 298105 & 8.708 & 2.434 .318 & $2,4 \%$ & 18.154 .473 & $1,8 \%$ & 92.970 .580 & $0,9 \%$ \\
\hline 3 & 298105 & 12.902 & 3.606 .776 & $3,5 \%$ & 19.845 .840 & $1,9 \%$ & 102.402 .249 & $1,0 \%$ \\
\hline 4 & 298105 & 15.367 & 4.295 .831 & $4,2 \%$ & 21.975 .954 & $2,1 \%$ & 114.317 .070 & $1,1 \%$ \\
\hline 5 & 298105 & 17.774 & 4.968 .704 & $4,8 \%$ & 24.764 .208 & $2,4 \%$ & 129.667 .634 & $1,3 \%$ \\
\hline 6 & 298105 & 20.908 & 5.844 .745 & $5,7 \%$ & 28.515 .620 & $2,8 \%$ & 150.737.394 & $1,5 \%$ \\
\hline 7 & 298105 & 25.235 & 7.054 .340 & $6,8 \%$ & 33.842 .167 & $3,3 \%$ & 180.325 .110 & $1,8 \%$ \\
\hline 8 & 298105 & 31.682 & 8.856 .399 & $8,6 \%$ & 42.273 .013 & $4,1 \%$ & 229.130 .312 & $2,2 \%$ \\
\hline 9 & 298110 & 44.915 & 12.555 .365 & $12,2 \%$ & 62.422 .419 & $6,1 \%$ & 327.488 .246 & $3,2 \%$ \\
\hline 10 & 300000 & 187.889 & 52.191 .364 & $51,0 \%$ & 253.337.914 & $24,8 \%$ & 1.121.121.994 & $11,0 \%$ \\
\hline Total & 2982950 & 368.461 & 10.293 .522 & $100,0 \%$ & 52.191 .364 & $51,0 \%$ & 253.337.914 & $24,8 \%$ \\
\hline Gini & & 0,6159 & & & 0,5515 & & 0,5183 & \\
\hline D10/D1 & & 61 & & & 294 & & 1.302 & \\
\hline
\end{tabular}

Fuente: DIAN. Cálculo de los autores. 


\section{Estimativos adicionales con base la integración de informaciones de la ENPH y la base de ingreso declarados ante la DIAN}

Es clara, entonces, la divergencia de resultados de concentración de ingresos a partir de la Encuesta nacional de presupuesto de los hogares publicada por el DANE y según las declaraciones de renta de personas naturales ante la DIAN. Para brindar mayor claridad, a continuación, se presentan dos estimativos adicionales sobre la concentración de ingresos. El primero de ellos se calcula al introducir en la base de la DIAN todos aquellos ingresos registrados por de la ENPH inferiores al ingreso base de liquidación del impuesto sobre la renta de personas naturales. De acuerdo con la tabla del impuesto sobre la renta de las personas naturales residentes en el país, se debe declarar a partir de los 1090 UVT, es decir, de los 3 millones 112.828 pesos por mes para 2017 .

El segundo estimativo se calcula al introducir toda la información de ingresos suministrada por la ENPH con la base de ingresos declarados por personas naturales ante la DIAN. En este caso, se supone que cada información de los encuestados correspondería a una persona natural, ya que la base de la DIAN se levanta a nivel de cada una de las personas naturales declarantes. No sobra señalar que en este caso existe una alta probabilidad de que algunos encuestados puedan encontrarse incluidos en la base de la DIAN, es decir que ya declaran el impuesto sobre la renta y complementarios.

Si bien la ENPH considera diferentes conceptos de ingreso, el que se aplicó a las declaraciones de renta y complementarios de la DIAN fue el total de ingresos monetarios y no monetarios, ya que se trata del mismo que utiliza el DANE para el cálculo del coeficiente de Gini a nivel nacional.

El primer estimativo brinda un índice de Gini de 0.6259 para las personas naturales del país en 2017, consecuente con una participación del decil 10 en el ingreso total de $51.3 \%$ y una relación de ingresos entre el decil 10 y el decil 1 de 96 veces. De igual manera, se observa que el ingreso promedio del decil 1 es de 535.416 pesos, mientras que el del decil 10 es de 51 millones 561.203 pesos (cuadro 11).

Cuadro 11. Incorporación de ingresos inferiores a 1090 UVT de la ENPH en la base de personas naturales declarantes la DIAN 2017

\begin{tabular}{|c|c|c|c|c|}
\hline Decil & $\begin{array}{c}\text { Número Personas } \\
\text { Naturales }\end{array}$ & $\begin{array}{c}\text { Ingreso Bruto } \\
\text { (MM\$) }\end{array}$ & $\begin{array}{c}\text { Ingreso Promedio } \\
\text { Mes (\$) }\end{array}$ & $\begin{array}{c}\text { Participación } \\
\text { en el Total }\end{array}$ \\
1 & 305312 & 1.962 & 535.416 & $0,5 \%$ \\
2 & 305349 & 7.925 & 2.162 .698 & $2,2 \%$ \\
3 & 305340 & 12.603 & 3.439 .622 & $3,4 \%$ \\
4 & 305332 & 15.396 & 4.202 .116 & $4,2 \%$ \\
5 & 305317 & 17.861 & 4.874 .885 & $4,8 \%$ \\
6 & 305348 & 21.042 & 5.742 .571 & $5,7 \%$ \\
7 & 305306 & 25.445 & 6.945 .127 & $6,9 \%$ \\
8 & 305717 & 32.053 & 8.737 .151 & $8,7 \%$ \\
9 & 304932 & 45.354 & 12.394 .542 & $12,3 \%$ \\
10 & 305328 & 188.917 & 51.561 .203 & $51,3 \%$ \\
Total & 3053281 & 368.557 & 10.059 .037 & $100,0 \%$ \\
Gini & & $\mathbf{0 , 6 2 5 6}$ & & \\
D10/D1 & & $\mathbf{9 6}$ & & \\
\hline
\end{tabular}

Fuente: DIAN y ENPH-DANE. Cálculo de los autores. 
Cuadro 12. Integración del total encuestados de la ENPH y de personas naturales declarantes ante DIAN 2017 (MM\$)

\begin{tabular}{|c|c|c|c|c|}
\hline Decil & $\begin{array}{c}\text { Número } \\
\text { Personas }\end{array}$ & $\begin{array}{c}\text { Ingreso Bruto } \\
\text { (MM\$) }\end{array}$ & $\begin{array}{c}\text { Ingreso Promedio } \\
\text { Mes (\$) }\end{array}$ & $\begin{array}{c}\text { Participación en } \\
\text { el Total }\end{array}$ \\
\hline 1 & 307353 & 2.848 & 772.266 & $0,8 \%$ \\
2 & 306678 & 8.207 & 2.230 .157 & $2,2 \%$ \\
3 & 307019 & 12.699 & 3.446 .936 & $3,4 \%$ \\
4 & 307047 & 15.476 & 4.200 .279 & $4,2 \%$ \\
5 & 307000 & 17.944 & 4.870 .918 & $4,8 \%$ \\
6 & 307006 & 21.131 & 5.735 .775 & $5,7 \%$ \\
7 & 307009 & 25.550 & 6.935 .151 & $6,9 \%$ \\
8 & 307017 & 32.131 & 8.721 .252 & $8,7 \%$ \\
9 & 307009 & 45.551 & 12.364 .217 & $12,3 \%$ \\
10 & 307013 & 189.310 & 51.384 .962 & $51,0 \%$ \\
Total & 3070151 & 370.849 & 10.065 .970 & $100,0 \%$ \\
Gini & & $\mathbf{0 , 6 2 0 2}$ & & \\
D10/D1 & & $\mathbf{6 6}$ & & \\
\hline
\end{tabular}

Fuente: DIAN y ENPH-DANE.

Este índice promedio Gini es ligeramente superior al respectivo según declaraciones de renta de personas naturales ante la DIAN en 2017: 0.6259 vs. 0.6159 .

El segundo estimativo da un índice de Gini de 0.6202, con una participación del decil 10 en el ingreso total del $51.0 \%$ y una relación entre ingresos del decil 10 y del decil 1 de 66 veces. Así mismo, se observa que el ingreso promedio del decil 1 es de 772.266 pesos, mientras que el del decil 10 es 51 millones 384.962 pesos (cuadro 12).

El índice promedio Gini de concentración para 2017 según los dos estimativos resulta muy similar: 0.6259 vs. 0.6202 , ostensiblemente mayores al correspondiente para el ingreso total según la ENPH: 0.5437, y relativamente cercanos al respectivo, de acuerdo con los ingresos brutos declarados por personas naturales ante la DIAN: 0.6159 .

\section{A manera de conclusión reflexiva}

De acuerdo con la ENPH del DANE durante el periodo 2016-, se observan:

1. Unos elevados índices Gini de concentración de ingresos totales de hogares que oscilan entre uno de 0.5437 , para el promedio nacional, en comparación con unos de 0.5303, para cabeceras municipales, y hasta uno de 0.4732 para centros poblados y rural disperso.

A su vez, el índice Gini de concentración de ingresos corrientes monetarios de hogares es claramente mayor que el de ingresos totales de hogares, al ser de 0.5789 para el promedio nacional, 0.5520 para cabeceras municipales y 0.5064 para centros poblados y rurales aislados (cuadro 2), en lo que se reitera el patrón diferencial a nivel territorial.

Con la característica adicional de que en el caso de los ingresos monetarios corrientes por trabajo de asalariados, como el principal componente de los ingresos monetarios corrientes (un 48.0\%, en promedio), el índice 
Gini es significativamente mayor hasta en niveles de $0.6240,0,5732$ y 0.6181 , respectivamente, consecuente con un agravamiento de la inequidad relativa en términos de los ingresos salariales de hogares.

Además, el índice de concentración del ingreso monetario corriente disponible a nivel nacional es relativamente superior al de las cabeceras y al de los centros poblados y el rural disperso: 0.6315 vs. 0.6113 y 0.6155 .

Así, entonces, se corroboraría por qué Colombia es quizás el segundo o tercer país en la región con mayor nivel de desigualdad de ingresos, además de constatarse que la desigualdad en cabeceras municipales es ostensiblemente mayor que en los centros rurales, en los que también subsisten mayores niveles de pobreza multidimensional, de informalidad y de precariedad institucional.

2. Al clasificar los hogares en cinco clases o conglomerados -alta, media, vulnerable, pobre y pobre extrema- el índice promedio de Gini es de 0.408 , en el caso de personas, y de 0.447 en el de hogares. Este menor nivel es explicado fundamentalmente por el menor grado de desagregación de la población respecto al de una desagregación por deciles -es decir, 5 en lugar de 10 agrupaciones-. He ahí la importancia del nivel de desagregación para la robustez del estimativo del índice de concentración.

Así mismo, al dividir en subdeciles la clase alta se encuentra que el índice de concentración Gini del ingreso es de 0.300, en tanto que el respectivo para la denominada clase media de 0.264 .

De otra parte, el índice de Gini de los ingresos totales para el decil 10 de hogares más ricos desagregándolo en 10 subdeciles -y para el 1\% de los hogares más ricos- subdecil 10 del decil 10 desagregado a su vez en 10 sub-deciles- es de 0.337 y 0.281 , respectivamente, claramente inferiores a los estimados de acuerdo con los ingresos declarados por personas naturales ante la DIAN en 2017.
Lo anterior, agravado por el hecho de que la participación del 1 por ciento y 1 por 1.000 de los hogares más ricos del país en el ingreso total de los hogares del país según la ENPH del periodo de 2016-2017 del DANE es ostensiblemente inferior a la equivalente para el caso de las personas naturales declarantes ante la DIAN en 2017, lleva a plantear un interrogante serio sobre el grado de representación de este tipo de hogares en la muestra de la ENPH, aparte del nivel de confiabilidad en los datos recolectados en una encuesta como la ENPH sin contrastación con otras fuentes fidedignas, en marcado contraste con el caso de las declaraciones de renta ante la DIAN. Es así como, por ejemplo, el número de hogares encuestados para la ENPH pertenecientes al $1 \%$ más rico fue de 655 , en comparación con 963 y 957 para hogares de los subdeciles 1 y 2 del decil 10.

De ahí la importancia de contrastar o complementar fuentes de información confiables para asegurar estimativos robustos sobre la concentración de ingresos. Innegablemente, la fuente más fidedigna para el conjunto de personas declarantes es la declarada ante la DIAN.

3. Al procederse a estimar los índices Gini de concentración con base exclusivamente en las declaraciones de renta de personas naturales ante la DIAN, se observa que el índice Gini de ingresos brutos alcanza el 0.6159 en 2017, algo que resulta sustancialmente mayor que el respectivo de ingresos totales, de acorde con la ENPH: 0.6159 vs. 0.5437 , pero similar al de ingresos corrientes disponibles, según la ENPH: 0.6159 vs. 0.6315 .

Aún más, el índice de concentración Gini de ingresos brutos de personas naturales entre los subdeciles del decil 10, con base la base de información de la DIAN, es de 0.5515 para 2017, significativamente superior al correspondiente según la ENPH -0.5515 vs. $0.337 \mathrm{y}$ algo inferior al índice promedio nacional entre los 10 deciles de ingreso. 
Con la característica adicional de que el índice Gini de concentración de ingresos brutos declarados ante la DIAN es de 0.5183 para el $1 \%$ de las personas naturales más ricas del país, el cual es inferior al índice Gini promedio nacional de ingresos totales, según la ENPH para 2017.

Ante estas circunstancias, resulta evidente que el estimativo de la concentración de ingresos resulta evidentemente más elevado con base en la información tributaria con sustento contrastable que con la de la encuesta de hogares sin debida contrastación de la veracidad de la información suministrada voluntariamente. Ello, aparte de problemas de representación de número de hogares encuestado a nivel de los hogares más ricos del país -el 1\% y 1 por 1.000, por ejemplo.

4. Como complemento, al incorporar los hogares de la ENPH que no están obligados a declarar renta ante la DIAN por tener unos ingresos brutos inferiores a $1090 \mathrm{UVT}$, a la base de la DIAN, se estima un índice de Gini de 0.6259 para las personas naturales del país en 2017, sustancialmente superior al correspondiente para los ingresos totales de hogares, según la ENPH: 0.6259 vs. 0.5437, pero ligeramente superior al respectivo según declaraciones de renta de personas naturales ante la DIAN en 2017: 0.6259 vs. 0.6159.

A su turno, al integrarse ambas bases de datos de la ENPH y la DIAN se encuentra que el índice de Gini de ingresos totales es de 0.6202, muy similar al anterior: 0.6259 vs. 0.6202 , pero ostensiblemente mayores al correspondiente para el ingreso total según la ENPH: 0.5437, y relativamente cercano al respectivo, de acuerdo con los ingresos brutos declarados por personas naturales ante la DIAN: 0.6159 .

Como consecuencia de todo lo anterior, se podría argumentar con suficientes elementos de juicio que el índice Gini de concentración de ingresos de personas naturales en Colombia en 2017 se habría ubicado en un rango entre 0.5437 y 0.6259 , con una elevada probabilidad de que se hubiera ubicado seguramente en los umbrales cercanos a 0.59-0.61.

La importancia de alcanzar un estimativo estadísticamente robusto sobre indicadores de concentración por tipos de ingreso y de contar con una base de información confiable y contrastable resulta crucial a la hora de servir como fuente de referencia básica para el diseño de políticas públicas de índole social y económica.

En este sentido, no sería aconsejable proceder con ese propósito con base exclusivamente de la ENPH sin una debida adecuación, complementación y contrastación con otras fuentes confiables y representativas como la de declaraciones de renta ante la DIAN.

Vale la pena resaltar en que la información fiscal es una "herramienta muy poderosa", no solo como fuente de información sobre la tributación, sino por la información adicional que brinda respecto a las fuentes de ingreso de las personas naturales como de la composición de su patrimonio, de tal forma que permite tomar "decisiones políticas y económicas" sobre una base más sólida. En este sentido, puede que esté pecando de "una fe excesiva en el poder de la información [fiscal] para generar grandes cambios transformadores en el terreno político" (Goldhammer, pp. 74-75), pero ello no niega que se trata de una herramienta indiscutiblemente útil para la toma de decisiones de política pública.

El análisis aquí desarrollado constituye una modesta ilustración de la problemática en cuestión, que podría servir para un debate necesario sobre mediciones de distribuciónconcentración de ingresos en el país. 


\section{Referencias}

DANE (2020). Metodología general Encuesta Nacional de Presupuestos de los Hogares - ENPH. Código: Dso-Enph-Met-001 Versión: 2 Fecha: 24/Feb/2020 Proceso: Procesos Misionales Operación o Investigación Estadística: ENPH - Encuesta Nacional. https://www.dane.gov.co/files/in vestigaciones/fichas/condiciones-de-vida/ DSO-ENPH-MET-001-V2.pdf

DANE. (2018). Metodología general Encuesta Nacional de Presupuestos de los Hogares -ENPH”. Código: DSO-ENPHFME-001. Versión:1.Fecha:2-Ago.-2018.https://www. dane.gov.co/files/investigaciones/fichas/ condiciones-de-vida/DSO-ENPH-MET001-V2.pdf

Derenoncourt, E. (2018). "Los orígenes históricos de la desigualdad global”. En: Delong, J. B. Boushey, H. y Steinbaum, M. (eds.) (2018). Debatiendo con Piketty. La agenda para la economía y la desigualdad. Barcelona: Ediciones Deusto.
Garay, L. J. y Espitia, J. E. (2019). Dinámica de las desigualdades en Colombia. Bogotá: Ediciones Desde Abajo.

Goldhammer, A. (2018). "El fenómeno Piketty”. En: Delong, J. B.,Boushey, H. y Steinbaum, M. (eds.) (2018). Debatiendo con Piketty. La agenda para la economía y la desigualdad. Ediciones Deusto.

Krugman, P. (2018). "Por qué estamos en la Nueva Edad Dorada”. En: Delong, J. B., Boushey, H. y Steinbaum, M. (eds.) (2018). Debatiendo con Piketty. La agenda para la economía y la desigualdad. Barcelona: Ediciones Deusto.

Tyson, L. y Spence, M (2018). "Explorar los efectos de la tecnología en la desigualdad de renta y riqueza”. En: Delong, J. B. Boushey, H. y Steinbaum, M. (eds.) (2018). Debatiendo con Piketty. La agenda para la economía y la desigualdad. Barcelona: Ediciones Deusto. 